\title{
Acoustic scattering by an inhomogeneous layer on a rigid plate
}

Article

Published Version

Zhang, B. and Chandler-Wilde, S. N. (1998) Acoustic scattering by an inhomogeneous layer on a rigid plate. SIAM Journal on Applied Mathematics (SIAP), 58 (6). pp. 19311950. ISSN 0036-1399 doi:

https://doi.org/10.1137/S0003613999631269 Available at https://centaur.reading.ac.uk/32654/

It is advisable to refer to the publisher's version if you intend to cite from the work. See Guidance on citing.

Published version at: http://dx.doi.org/10.1137/S0003613999631269

To link to this article DOI: http://dx.doi.org/10.1137/S0003613999631269

Publisher: Society for Industrial and Applied Mathematics

All outputs in CentAUR are protected by Intellectual Property Rights law, including copyright law. Copyright and IPR is retained by the creators or other copyright holders. Terms and conditions for use of this material are defined in the End User Agreement.

\section{www.reading.ac.uk/centaur}

\section{CentAUR}

Central Archive at the University of Reading 
Reading's research outputs online 


\title{
ACOUSTIC SCATTERING BY AN INHOMOGENEOUS LAYER ON A RIGID PLATE*
}

\author{
BO ZHANG ${ }^{\dagger}$ AND SIMON N. CHANDLER-WILDE ${ }^{\dagger}$
}

\begin{abstract}
The problem of scattering of time-harmonic acoustic waves by an inhomogeneous fluid layer on a rigid plate in $\mathbf{R}^{2}$ is considered. The density is assumed to be unity in the media: within the layer the sound speed is assumed to be an arbitrary bounded measurable function. The problem is modelled by the reduced wave equation with variable wavenumber in the layer and a Neumann condition on the plate. To formulate the problem and prove uniqueness of solution a radiation condition appropriate for scattering by infinite rough surfaces is introduced, a generalization of the Rayleigh expansion condition for diffraction gratings. With the help of the radiation condition the problem is reformulated as a system of two second kind integral equations over the layer and the plate. Under additional assumptions on the wavenumber in the layer, uniqueness of solution is proved and the nonexistence of guided wave solutions of the homogeneous problem established. General results on the solvability of systems of integral equations on unbounded domains are used to establish existence and continuous dependence in a weighted norm of the solution on the given data.
\end{abstract}

Key words. radiation condition, rough surface scattering, inhomogeneous layer, integral equations, existence, uniqueness, guided waves, Neumann condition

AMS subject classifications. 35B40, 35L05

PII. S00036139996312697

1. Introduction. Consider the scattering problem of a time-harmonic acoustic plane wave incident to an inhomogeneous fluid layer sitting on a rigid plate in $\mathbf{R}^{2}$. The density is assumed to be unity in the media, and the sound speed is supposed an arbitrary bounded measurable function in the layer and a constant above the layer, so that the medium, above the layer, is homogeneous. In this paper an integral equation method is used to study the existence of a unique solution to the problem, in particular for the case where the inhomogeneity extends to infinity without decaying. A radiation condition appropriate for scattering by infinite one-dimensional rough surfaces is introduced, which is a generalization of the standard radiation condition used in the study of plane wave diffraction by one-dimensional periodic gratings (see $[2,3,20,21])$. The problem is then reformulated as an equivalent system of two second kind integral equations, over the infinite layer and the plate, respectively. Uniqueness of solution is proved, and then existence of solution is obtained by applying a form of Fredholm alternative based on general results on the solvability of systems of integral equations on unbounded domains [9].

Integral equation methods have been widely used in the theoretical and numerical study of wave scattering by finite obstacles or local inhomogeneities (see, e.g., [13, 14] and the references quoted there). More recently they have been employed to study scattering by periodic structures $[12,18,20,21,22]$ and by a nonstratified local inhomogeneity in a stratified medium [26]. Integral equation formulations have also been used extensively in computations of plane wave scattering by infinite one-dimensional rough surfaces and interfaces (see, e.g., [15]), but little attention appears to have

\footnotetext{
* Received by the editors December 2, 1996; accepted for publication (in revised form) July 18, 1997; published electronically August 18, 1998. This research was supported by the UK Engineering and Physical Sciences Research Council under grant GR/K24406.

http://www.siam.org/journals/siap/58-6/31269.html

${ }^{\dagger}$ Department of Mathematics and Statistics, Brunel University, Uxbridge UB8 3PH, UK (bo.zhang@brunel.ac.uk, simon.chandler-wilde@brunel.ac.uk).
} 
been paid in the literature to their mathematical justification (a recent exception is [16]). This present paper is intended as a contribution to the mathematical analysis of such problems. It is related, in terms of results and methods of argument, to recent studies of scattering by an inhomogeneous impedance half-plane in a homogeneous medium [4]; by a one-dimensional rough surface which is small perturbation of a flat half-plane [5, 7]; and of electromagnetic waves by an inhomogeneous conducting or dielectric layer on a perfectly conducting plate [6]. In particular, this paper is an extension of the study in [6] for the Dirichlet case to that of the Neumann case. Related work on plane wave scattering by a periodic inhomogeneous layer is contained in $[21,25]$.

The outline of this paper is as follows. In the next section the scattering problem is introduced and the radiation condition discussed. Section 3 is devoted to the derivation of an integral equation formulation which is equivalent to the scattering problem. In section 4 , under assumptions on the wavenumber variation in the layer, we establish a basic inequality satisfied by the solution. Using this inequality, results from [9] which are summarized in Appendix A, a key lemma from [7], and extensions of arguments in [6], general uniqueness and existence results are established in sections 5 and 6 , respectively. In particular the results apply to any bounded space variation of the wavenumber, provided the real part of the square of the wavenumber is everywhere nondecreasing in the $x_{2}$ direction and has some minimum net increase over the depth of the layer. Our uniqueness results also establish (by virtue of results in Appendix B) that, under these same conditions, guided wave solutions of the homogeneous problem are not supported by the inhomogeneous layer.

We conclude this section by introducing some notations. For $h \in \mathbf{R}$, define $\Gamma_{h}=\left\{x \in \mathbf{R}^{2} \mid x_{2}=h\right\}$ and $U_{h}=\left\{x \in \mathbf{R}^{2} \mid x_{2}>h\right\}$. We write $U$ and $\Gamma$ for $U_{0}$ and $\Gamma_{0}$, respectively. We also write $E_{h}=U \backslash \overline{U_{h}}$ for $h>0$ and define $D_{A}=\left\{x \in \mathbf{R}^{2}|| x_{1} \mid<A\right\}$, $A>0$, and $\Gamma_{h}(A)=\Gamma_{h} \cap D_{A}, E_{h}(A)=E_{h} \cap D_{A}$. For $v \in L_{\infty}(U)$ denote by $\partial_{j} v$, $j=1,2$, the (distributional) derivative $\partial v(x) / \partial x_{j}$. Finally, for $A>0, x \in \mathbf{R}^{2}$, let $B_{A}(x)=\left\{y \in \mathbf{R}^{2}|| y-x \mid<A\right\}$.

2. The scattering problem and radiation condition. Suppose that the halfplane $U$ is filled with an inhomogeneous acoustic medium of (possibly complex) sound speed $c(x)$ and density unity. Let $k$ denote the wavenumber of the medium. Then $k(x)=\omega / c(x)$, where $\omega>0$ is the angular frequency, and the time-harmonic acoustic wave propagation (with time dependence $\exp (-i \omega t)$ suppressed) is governed by the reduced wave equation

$$
\Delta u+k^{2} u=0 \quad \text { in } \quad U
$$

Suppose also that the inhomogeneous medium has a rigid boundary. Then the Neumann boundary condition is satisfied at the boundary:

$$
\frac{\partial u}{\partial n}=0 \quad \text { on } \quad \Gamma
$$

where $n$ is the unit normal at $\Gamma$ directed out of $U$.

We assume that the wavenumber $k$ satisfies that $\Re k, \Im k \geq 0$, so that $\Im\left(k^{2}\right) \geq 0$. We make additionally throughout the following assumptions on $k$, the second of which implies that the medium is homogeneous above some finite layer $E_{B}$.

(A1) $k \in L_{\infty}(U)$.

(A2) There are two positive constants $B$ and $k_{+}$such that $k(x)=k_{+}$for all $x \in U_{B}$. 
These two assumptions are sufficient (together with the radiation condition we introduce below) to derive, in section 3 , an equivalent integral equation formulation of the problem. In sections 4 and 5 we address the question of uniqueness of solution which is related to the question of existence or otherwise of guided wave solutions of the homogeneous problem.

We remark that the radiation condition we will impose will ensure that the scattered field does not contain a downwards propagating component, but (in common with the usual radiation condition for plane wave incidence on periodic gratings) will not rule out guided waves localized in the inhomogeneous layer (see Theorem B.2 in Appendix B, where a precise definition of a guided wave in this context is given). Thus, to prove any uniqueness result, we will have to impose additional conditions (on $k$ ) which rule out guided waves. In other words (and more positively), any uniqueness proof will simultaneously establish conditions for the nonexistence of guided waves.

The additional assumptions we require for our uniqueness proof (sections 4 and 5 ) and to prove existence of solution (section 6) are that Assumption (A3) or Assumptions (A4) and (A5) below are satisfied.

(A3) There is a positive constant $\lambda_{1}$ such that $\Im\left(k^{2}(x)\right) \geq \lambda_{1}$ for almost all $x \in E_{B}$.

(A4) $\Re\left(k^{2}(x)\right)$ is monotonic increasing as $x_{2}$ increases; precisely,

$$
\operatorname{ess}_{\inf _{x \in U}} \Re\left[k^{2}\left(x+e_{2} h\right)-k^{2}(x)\right] \geq 0
$$

for all $h>0$, where $e_{2}=(0,1)$.

(A5) There are positive constants $\lambda_{2}, d$ with $d<B$ such that

$$
\operatorname{ess} \sup _{x \in E_{d}} \Re\left[k^{2}(x)\right] \leq k_{+}^{2}-\lambda_{2} \text {. }
$$

Assumption (A3) is a requirement that the layer $E_{B}$ is lossy (energy absorbing). In the case when $k$ is real, Assumption (A4) is a requirement that in the physical problem the wave speed $c(x)$ is nonincreasing as $x_{2}$ increases, and (A5) requires, additionally, some minimum net decrease in the wave speed over the depth of the layer.

Regarding the practical relevance of the above problem, we remark that this configuration has been used as a model of outdoor sound propagation over level terrain in the presence of wind gradients. In this context it is usual to model the ground surface as a porous half-space or layer and common to represent its effect by an impedance or Robin boundary condition (see, e.g., $[1,23,24]$ ): in the limiting case of high flow resistivity of the porous medium or low frequency of the incident wave, this condition reduces to the Neumann condition (2). The case when $c$ decreases with height corresponds to the case of downwind propagation. Of course, it is well known, for the case of a purely vertical stratification $\left(c(x)\right.$ depending only on $\left.x_{2}\right)$, that guided waves are not supported when $c$ decreases with height. Our uniqueness results will show that this remains true when horizontal variations in $c(x)$ are also permitted.

We remark that in the Dirichlet boundary condition case, when (2) is replaced by the condition $u=0$ on $\Gamma$, the assumption (A4) is sufficient to ensure uniqueness as demonstrated in [6]. In the Neumann boundary condition case the radiation condition (3) below and (A4) are not together sufficient to guarantee a unique solution as is shown by the simple example $u(x)=\exp \left( \pm i k_{+} x_{1}\right)$, which satisfies (1)-(2) with $k \equiv k_{+}$ and, by Remark 2.3 below, the radiation condition (3).

Let $u^{\mathrm{i}}(x)=\exp \left(i k_{+} x \cdot \alpha\right)$ be the time-harmonic incoming plane wave, incident from $U_{B}$ on the finite inhomogeneous layer $E_{B}$, where $x \in \mathbf{R}^{2}, \alpha=(\cos \theta,-\sin \theta) \in$ 
$\mathbf{R}^{2}$, and $\theta \in(0, \pi)$ is the incident angle. We are interested in finding the total field $u$ satisfying the reduced wave equation (1) and the Neumann condition (2).

In order to determine the physical solution $u$, a radiation condition as $x_{2}$ tends to infinity has to be imposed on the scattered field $u^{\mathrm{s}}=u-u^{\mathrm{i}}$, that is, the scattered field $u^{\mathrm{s}}$ should behave as an outgoing wave as $x_{2} \rightarrow \infty$. The standard Sommerfeld radiation condition is not appropriate in this context as we cannot expect that $u^{\mathrm{s}}$ will decay in the $x_{1}$ direction. We will use the radiation condition proposed in [3] (see also [4] and [6]), which we will refer to as the upward propagating radiation condition (UPRC), and will usefully relate this condition to the Sommerfeld radiation condition below. To this end introduce the following definitions.

Definition 2.1. Given a domain $G \subset \mathbf{R}^{2}$, call $v \in C^{2}(G) \cap L_{\infty}(G)$ a radiating solution of the Helmholtz equation in $G$ if $\Delta v+k_{+}^{2} v=0$ in $G$ and

$$
\begin{array}{r}
v(x)=O\left(r^{-1 / 2}\right), \\
\frac{\partial v(x)}{\partial r}-i k_{+} v(x)=o\left(r^{-1 / 2}\right)
\end{array}
$$

as $r=|x| \rightarrow \infty$, uniformly in $x /|x|$.

Let $\Phi(x, y)$ denote the free-space Green's function for $\Delta+k_{+}^{2}$, that is,

$$
\Phi(x, y)=\frac{i}{4} H_{0}^{(1)}\left(k_{+}|x-y|\right), \quad x, y \in \mathbf{R}^{2}, \quad x \neq y,
$$

where $H_{0}^{(1)}$ is the Hankel function of the first kind of order zero.

Definition 2.2. Given a domain $G \subset \mathbf{R}^{2}$, say that $v: G \rightarrow \mathbf{C}$ satisfies the UPRC in $G$ if, for some $h \in \mathbf{R}$ and $\phi \in L_{\infty}\left(\Gamma_{h}\right), U_{h} \subset G$ and

$$
v(x)=2 \int_{\Gamma_{h}} \frac{\partial \Phi(x, y)}{\partial y_{2}} \phi(y) d s(y), \quad x \in U_{h} .
$$

Note that the existence of the integral in (3) for arbitrary $\phi \in L_{\infty}\left(\Gamma_{h}\right)$ is assured by the bound which follows from the asymptotic behavior of the Hankel function for small and large argument,

$$
\left|\frac{\partial \Phi(x, y)}{\partial y_{2}}\right| \leq C\left|x_{2}-y_{2}\right|\left(|x-y|^{-2}+|x-y|^{-3 / 2}\right), \quad x, y \in \mathbf{R}^{2}, x \neq y,
$$

which holds for some constant $C>0$ dependent only on $k_{+}$.

The following result explores properties of the upward propagating radiation condition and, in particular, shows that any radiating solution satisfies the UPRC.

Lemma 2.3. Given $H \in \mathbf{R}$ and $v: U_{H} \rightarrow \mathbf{C}$, the following statements are equivalent:

(i) $v \in C^{2}\left(U_{H}\right), v \in L_{\infty}\left(U_{H} \backslash U_{a}\right)$ for all $a>H, \Delta v+k_{+}^{2} v=0$ in $U_{H}$, and $v$ satisfies the UPRC in $U_{H}$;

(ii) there exists a sequence $\left(v_{n}\right)$ of radiating solutions such that $v_{n}(x) \rightarrow v(x)$ uniformly on compact subsets of $U_{H}$ and

$$
\sup _{x \in U_{H} \backslash U_{a}, n \in \mathbf{N}}\left|v_{n}(x)\right|<\infty
$$

for all $a>H$;

(iii) $v$ satisfies (3) for $h=H$ and some $\phi \in L_{\infty}\left(\Gamma_{H}\right)$; 
(iv) $v \in L_{\infty}\left(U_{H} \backslash U_{a}\right)$ for some $a>H$ and $v$ satisfies (3) for each $h>H$, with $\phi=\left.v\right|_{\Gamma_{h}}$, the restriction of $v$ to $\Gamma_{h}$;

(v) $v \in C^{2}\left(U_{H}\right), v \in L_{\infty}\left(U_{H} \backslash U_{a}\right)$ for all $a>H, \Delta v+k_{+}^{2} v=0$ in $U_{H}$, and, for every $h>H$ and radiating solution in $U_{H}, w$, such that the restrictions of $w$ and $\partial_{2} w$ to $\Gamma_{h}$ are in $L_{1}\left(\Gamma_{h}\right)$, it holds that

$$
\int_{\Gamma_{h}}\left(v \frac{\partial w}{\partial n}-w \frac{\partial v}{\partial n}\right) d s=0
$$

This lemma was proved as Theorem 2.1 in [6], using Green's second theorem and the local regularity estimate contained in the next lemma (see [19, Theorem 3.9 and Lemma 4.1]) which is also needed later.

Lemma 2.4. If $G \subset \mathbf{R}^{2}$ is open and bounded, $v \in L_{\infty}(G)$, and $\Delta v=f \in L_{\infty}(G)$ (in a distributional sense) then $v \in C^{1}(G)$ and

$$
|\nabla v(x)| \leq C(d(x))^{-1}\left(\|v\|_{\infty}+\left\|d^{2} f\right\|_{\infty}\right), \quad x \in G,
$$

where $C$ is an absolute constant and $d(x)=\operatorname{dist}(x, \partial G)$.

Remark 2.1. A consequence of Lemma 2.4 is that if $\Delta v+k^{2} v=0$ in some region $G$ and $v \in L_{\infty}(G), k \in L_{\infty}(G)$, then $v \in C^{1}(G)$ and $\nabla v$ is bounded in every compact subset of $G$. Further, if the sequence $\left(v_{n}\right) \subset L_{\infty}(G)$ is uniformly bounded, $\Delta v_{n}+$ $k_{*}^{2} v_{n}=0$ in $G$ for some $k_{*} \in \mathbf{C}$ and each $n$, and $v_{n}(x) \rightarrow v(x)$ uniformly on compact subsets of $G$, then $v \in C^{2}(G)$ and $\Delta v+k_{*}^{2} v=0$ in $G$.

Let $\mathcal{R}(U)=\left\{v \in C(\bar{U}) \cap C^{1}(U) \mid \partial_{2} v \in C(\bar{U})\right\}$. Then our problem of scattering of a time-harmonic plane wave by an inhomogeneous layer can now be formulated as the following boundary value problem.

Problem $(P)$ : Find $u \in \mathcal{R}(U)$ such that (i) $u$ satisfies the reduced wave equation (1) in a distributional sense and the Neumann condition (2); (ii) $u^{\mathrm{s}}$ satisfies the upward propagating radiation condition (3); and (iii) $u$ is bounded in $E_{A}$ for every $A>0$.

Remark 2.2. From (iii), Lemma 2.4, and a reflection argument based on the Neumann condition (2), it follows that $u \in C^{1}(\bar{U}) \cap C^{2}\left(U_{B}\right)$ and that

$$
\sup _{x \in E_{A}}[|\nabla u(x)|+|u(x)|]<\infty
$$

for every $A>0$. By (1), the same reflection principle, and standard local regularity results [19], we have also that $u \in H_{\mathrm{loc}}^{2}(U)$.

Remark 2.3. The radiation condition (3) is a generalization of the standard radiation condition for one-dimensional periodic gratings. Precisely, it was proved in [3] that if $u^{\mathrm{s}}$ has the usual representation as a Rayleigh expansion $[2,20,21]$ in some $U_{b}$ then it also satisfies (3) for all $h>b$ and so satisfies the UPRC. As a consequence any upward or horizontally propagating plane wave satisfies the UPRC. Thus, in the case $k \equiv k_{+}, u=u^{\mathrm{i}}+u^{\mathrm{r}}$ is a solution of problem (P) with the Neumann boundary condition (2) replaced by the impedance condition

$$
\partial_{2} u+i k_{+} u=0, \quad x \in \Gamma
$$

where $u^{\mathrm{r}}$ is the reflected wave $u^{\mathrm{r}}(x)=\beta \exp \left(i k_{+} x \cdot \alpha^{\prime}\right)$, with $\alpha^{\prime}=(\cos \theta, \sin \theta)$ and $\beta=(\sin \theta-1) /(\sin \theta+1)$.

3. An integral equation formulation. In order to derive an equivalent integral equation formulation for problem $(\mathrm{P})$, define

$$
G(x, y):=\Phi(x, y)+\Phi\left(x, y^{\prime}\right)+P\left(x-y^{\prime}\right), \quad x, y \in \bar{U}, \quad x \neq y,
$$


where, for $x \in \bar{U}, x^{\prime}=\left(x_{1},-x_{2}\right)$, and

$$
P(x):=-\frac{i k_{+}}{2 \pi} \int_{-\infty}^{\infty} \frac{\exp \left(i\left[x_{1} s+x_{2} \sqrt{k_{+}^{2}-s^{2}}\right]\right)}{\sqrt{k_{+}^{2}-s^{2}}\left(\sqrt{k_{+}^{2}-s^{2}}+k_{+}\right)} d s
$$

with $\Im \sqrt{k_{+}^{2}-s^{2}} \geq 0$. It is shown in [10] (or see [4]) that $P \in C(\bar{U}) \cap C^{\infty}(\bar{U} \backslash\{0\}$ ) and that $P$ is a radiating solution of the Helmholtz equation in $U$. Further [10], $\partial P(x) / \partial x_{1}$ is bounded in $\bar{U} \backslash\{0\}$ and (see [11, equation (39)]) $\partial P(x) / \partial x_{2}$ satisfies

$$
\frac{\partial P(x)}{\partial x_{2}}+i k_{+} G(0, x)=0, \quad x \in \bar{U}, \quad x \neq 0 .
$$

Methods for computing $P$ and its gradient efficiently are developed in [11].

From the above equations and properties (see [10] or [4] for further detail) it follows that $G$ is the impedance Green's function for the Helmholtz equation in $U$ and in particular that

$$
\Delta_{x} G(x, y)+k_{+}^{2} G(x, y)=0, \quad x, y \in U, \quad x \neq y,
$$

and

$$
\frac{\partial G}{\partial x_{2}}(x, y)+i k_{+} G(x, y)=0, \quad x \in \Gamma, \quad y \in U, \quad x \neq y .
$$

An important property of $G$ is its rapid asymptotic decay rate as $|x-y| \rightarrow \infty$ with $x$ and $y$ close to the boundary $\Gamma$. In particular, it follows from [5, Lemma 3.1] that, for some constant $C$ depending only on $k_{+}$,

$$
|G(x, y)|, \quad\left|\nabla_{x} G(x, y)\right|, \quad\left|\nabla_{y} G(x, y)\right| \leq C \frac{\left(1+x_{2}\right)\left(1+y_{2}\right)}{|x-y|^{3 / 2}},
$$

for all $x, y \in U$ with $|x-y| \geq 1$. On the other hand, from the definition of $G$, asymptotic properties of the Hankel function and the above properties of $P$, it follows that

$$
|G(x, y)| \leq C(1+|\log | x-y||), \quad\left|\nabla_{x} G(x, y)\right|, \quad\left|\nabla_{y} G(x, y)\right| \leq C|x-y|^{-1},
$$

for all $x, y \in U$ with $|x-y| \leq 1$. From (13) and (14) it follows that, for $y \in E_{B}$, $x_{1} \in \mathbf{R}, x_{2}=a>B$,

$$
|G(x, y)|, \quad\left|\nabla_{x} G(x, y)\right|, \quad\left|\nabla_{y} G(x, y)\right| \leq C_{a}\left(1+\left|x_{1}-y_{1}\right|\right)^{-3 / 2},
$$

where $C_{a}$ is a positive constant depending only on $a, B$, and $k_{+}$.

Before establishing the integral equation formulation in Theorem 3.3 below we first investigate properties of volume and surface potentials of the type appearing in (17).

Lemma 3.1. Define the volume potential $v$ with density $\phi \in L_{\infty}\left(E_{B}\right)$ by

$$
v(x)=\int_{E_{B}} G(x, y) \phi(y) d y, \quad x \in \bar{U},
$$


and extend the definition of $\phi$ to $U$ by setting $\phi(x)=0, x \in U_{B}$. Then $v \in C^{1}(\bar{U}) \cap$ $L_{\infty}\left(E_{a}\right)$ for $a>0, \partial_{2} v+i k_{+} v=0$ on $\Gamma, \Delta v+k_{+}^{2} v=-\phi$ in $U$, and $v$ satisfies the UPRC.

Proof. By making use of the bounds (13) and (14), and the differentiability of $G(x, y)$ for $x \neq y$, together with the dominated convergence theorem, it can easily be shown that $v \in C^{1}(\bar{U}) \cap L_{\infty}\left(E_{a}\right), a>0$, and, on noting (12), that $\partial_{2} v+i k_{+} v=0$ on $\Gamma$. For $n \in \mathbf{N}$ define

$$
v_{n}(x)=\int_{E_{B}(n)} G(x, y) \phi(y) d y, \quad x \in \bar{U} .
$$

Given an arbitrary $A>0$ choose the positive integer $M \in \mathbf{N}$ so that $M>A$. Then from standard results on volume potentials [13], in $D_{A} \cap U$ it holds that $\Delta v_{M}+$ $k_{+}^{2} v_{M}=-\phi$ and $\left(\Delta+k_{+}^{2}\right)\left(v_{n}-v_{M}\right)=0$, for $n=M+1, M+2, \ldots$ Further, $v_{n}(x) \rightarrow v(x)$ as $n \rightarrow \infty$, uniformly on compact subsets of $D_{A}$, so that, by Remark $2.1,\left(\Delta+k_{+}^{2}\right)\left(v-v_{M}\right)=0$ in $D_{A}$. Since $A>0$ is arbitrary, we have shown that $\Delta v+k_{+}^{2} v=-\phi$ in $U$. Further, for $n=1,2, \ldots, v_{n}$ is a radiating solution in $U_{B}$ and, using the bounds (13) and (14), it is easily seen that (5) is satisfied for all $a>0$. It follows from the equivalence of (i) and (ii) in Lemma 2.3 that $v$ satisfies the UPRC. The proof is complete.

Lemma 3.2. Define the single layer potential $S$ with density $\psi \in B C(\Gamma)$ by

$$
S(x)=\int_{\Gamma} G(x, y) \psi(y) d s(y), \quad x \in \bar{U} .
$$

Then $S \in \mathcal{R}(U) \cap L_{\infty}\left(E_{a}\right)$ for $a>0, \partial_{2} S+i k_{+} S=-\psi$ on $\Gamma, \Delta S+k_{+}^{2} S=0$ in $U$, and $S$ satisfies the UPRC.

Proof. By means of the bounds (13) and (14), and the differentiability of $G(x, y)$ for $x \neq y$, together with the dominated convergence theorem, it is easy to show that $S \in C(\bar{U}) \cap C^{1}(U) \cap L_{\infty}\left(E_{a}\right), a>0$. To see that $S$ satisfies the Helmholtz equation, let us define, for $n \in \mathbf{N}$,

$$
S_{n}(x)=\int_{\Gamma(n)} G(x, y) \psi(y) d s(y), \quad x \in \bar{U} .
$$

Then $S_{n} \in C^{2}(U)$ and $\left(\Delta+k_{+}^{2}\right) S_{n}=0$ in $U$. Further, $S_{n}(x) \rightarrow S(x)$ as $n \rightarrow \infty$, uniformly on compact subsets of $U$, so that, by Remark 2.1, $S \in C^{2}(U)$ and $(\Delta+$ $\left.k_{+}^{2}\right) S=0$ in $U$. Moreover, for $n=1,2, \ldots, S_{n}$ is a radiating solution in $U$ and, using the bounds (13) and (14), it is easily seen that (5) is satisfied for all $a>0$. It follows from the equivalence of (i) and (ii) in Lemma 2.3 that $S$ satisfies the UPRC.

From (10) it follows that, for $x \in U$ and $y \in \Gamma$,

$$
\frac{\partial G(x, y)}{\partial x_{2}}=2 \frac{\partial \Phi(x, y)}{\partial x_{2}}+\frac{\partial P\left(x-y^{\prime}\right)}{\partial x_{2}}=-2 \frac{\partial \Phi(x, y)}{\partial y_{2}}-i k_{+} G(x, y) .
$$

By making use of (16), the bounds (13) and (14), the dominated convergence theorem, and the jump relation for standard double-layer potentials [13, Theorem 2.13], it can be easily derived that $\partial_{2} S \in C(\bar{U})$ and $\partial_{2} S+i k_{+} S=-\psi$ on $\Gamma$. The proof is thus complete.

THEOREM 3.3. Let $u$ be a solution of problem $(P)$. Then we have

$$
\begin{gathered}
u(x)=u^{\mathrm{i}}(x)+u^{\mathrm{r}}(x)+\int_{E_{B}} u(y)\left[k^{2}(y)-k_{+}^{2}\right] G(x, y) d y \\
-i k_{+} \int_{\Gamma} u(y) G(x, y) d s(y), \quad x \in \bar{U} .
\end{gathered}
$$


Proof. Take $x \in U$, choose $a>\max \left(x_{2}, B\right), A>\left|x_{1}\right|$, and $\epsilon>0$ sufficiently small and apply Green's second theorem to $G(x, \cdot)$ and $u$ in the bounded region $E_{a}(A) \backslash \overline{B_{\epsilon}(x)}$, and then let $\epsilon \rightarrow 0$ to obtain that

$$
\begin{aligned}
u(x)= & \int_{E_{B}(A)} u(y)\left[k^{2}(y)-k_{+}^{2}\right] G(x, y) d y \\
& +\int_{\partial\left(E_{a}(A)\right)}\left[G(x, y) \frac{\partial u}{\partial n}(y)-u(y) \frac{\partial G(x, y)}{\partial n(y)}\right] d s(y) .
\end{aligned}
$$

Letting $A \rightarrow \infty$ in (18), in view of (13), (2), and (12), we find that

$$
u(x)=\int_{E_{B}} u(y)\left[k^{2}(y)-k_{+}^{2}\right] G(x, y) d y-i k_{+} \int_{\Gamma} u(y) G(x, y) d s(y)+I_{a},
$$

where

$$
I_{a}=\int_{\Gamma_{a}}\left[G(x, y) \frac{\partial u}{\partial n}(y)-u(y) \frac{\partial G(x, y)}{\partial n(y)}\right] d s(y) .
$$

Recall that, by Remark 2.3, $u^{\mathrm{i}}+u^{\mathrm{r}}$ is a solution of problem $(\mathrm{P})$ with $k \equiv k_{+}$and the Neumann condition (2) replaced by the impedance condition (8). By the same argument as used to derive (19) we can show that $u^{\mathrm{i}}(x)+u^{\mathrm{r}}(x)=\tilde{I}_{a}$, where $\tilde{I}_{a}$ is given by (20) but with $u$ replaced by $u^{\mathrm{i}}+u^{\mathrm{r}}$. It follows that

$$
I_{a}=u^{\mathrm{i}}(x)+u^{\mathrm{r}}(x)+\int_{\Gamma_{a}}\left[G(x, y) \frac{\partial w}{\partial n}(y)-w(y) \frac{\partial G(x, y)}{\partial n(y)}\right] d s(y),
$$

where $w=u^{\mathrm{s}}-u^{\mathrm{r}}$. Further, $w$ satisfies the UPRC and $G(x, \cdot)$ is a radiating solution in $U_{b}$ for $b>x_{2}$ so that, in view of (13) and the equivalence of (i) and (v) in Lemma 2.3, the integral in (21) vanishes. Thus (17) follows for $x \in U$. That (17) holds also for $x \in \Gamma$ follows from the continuity in $\bar{U}$ of $u, u^{\mathrm{i}}, u^{\mathrm{r}}$, and of the volume and surface potentials appearing in (17).

From Remark 2.3 and Lemmas 3.1 and 3.2 we have the following converse result.

THEOREM 3.4. If $u$ satisfies (17) and $u \in B C\left(\overline{E_{B}}\right)$, then $u$ satisfies problem $(\mathrm{P})$.

Remark 3.1. From Theorems 3.3 and 3.4 it follows that problem $(\mathrm{P})$ is equivalent to the integral equation (17).

4. A basic inequality. In this section we prove a basic inequality satisfied by the solution of the problem (1)-(2) which plays an essential role in the proof of the uniqueness theorem.

Suppose that $u \in \mathcal{R}(U)$ satisfies (1) and (2). Then, by Remark 2.2, $u \in C^{1}(\bar{U}) \cap$ $H_{\mathrm{loc}}^{2}(U)$. Let $a>B$ and define, for $A>0$,

$$
\begin{array}{ll}
J_{A}=\Im \int_{\Gamma_{a}(A)} \bar{u} \partial_{2} u d s, & I_{A}=\int_{\Gamma_{a}(A)}\left\{\left|\partial_{2} u\right|^{2}-\left|\partial_{1} u\right|^{2}+k_{+}^{2}|u|^{2}\right\} d s, \\
L_{A}=\Re \int_{\Gamma_{a}(A)} \bar{u} \partial_{2} u d s, & K_{A}=\int_{E_{B}(A)}|u|^{2} d x+\int_{\Gamma(A)}|u|^{2} d s .
\end{array}
$$

For $b \in \mathbf{R}$, let $\gamma(b)=\left\{\left(b, x_{2}\right) \mid 0 \leq x_{2} \leq a\right\}$.

TheOrem 4.1. Assume that (A3) holds or that both (A4) and (A5) hold. Then, for some nonnegative constants $C_{j}, j=1,2,3,4,5$, there holds

$$
K_{A} \leq C_{1} I_{A}+C_{2} L_{A}-C_{3} J_{A}+C_{4} R_{1}(A)+C_{5} R_{2}(A)
$$


for all $A>0$, where

$$
R_{1}(A)=\left[\int_{\gamma(A)}+\int_{\gamma(-A)}\right]\left|\bar{u} \partial_{1} u\right| d s
$$

and

$$
R_{2}(A)=\Re\left[\int_{\gamma(A)}-\int_{\gamma(-A)}\right]\left(2 x_{2} \partial_{2} \bar{u} \partial_{1} u+\bar{u} \partial_{1} u\right) d s .
$$

Proof. We will deduce the inequality (24) first of all in the case that (A3) holds.

Apply Green's first theorem to $u$ and $\bar{u}$ in $E_{a}(A)$, and take the imaginary part of the result thus obtained to get, since $\Im\left(k^{2}(x)\right)=0$ for $x_{2}>B$ and $\partial u / \partial n=0$ on $\Gamma$,

$$
\int_{E_{B}(A)} \Im\left(k^{2}\right)|u|^{2} d x+J_{A} \leq R_{1}(A) .
$$

Let $\eta \in C^{1}[0, \infty)$ be such that $0 \leq \eta(t) \leq 1$ for $t \geq 0, \eta(t)=1$ for $0 \leq t \leq B / 2$, $\eta(t)=0$ for $t \geq B$, and $\left|\eta^{\prime}(t)\right| \leq C \eta^{1 / 2}(t)$ for $t \geq 0$ and some constant $C$. Then, by applying Green's first theorem to $u$ and $\eta\left(x_{2}\right) \bar{u}$ in $E_{B}(A)$, we obtain that

$$
\begin{aligned}
\int_{E_{B}(A)} \eta\left(x_{2}\right)|\nabla u|^{2} d x \leq & \int_{E_{B}(A)}|k|^{2} \eta\left(x_{2}\right)|u|^{2} d x \\
& +\int_{E_{B}(A)}\left|\eta^{\prime}\left(x_{2}\right) \bar{u} \partial_{2} u\right| d x+R_{1}(A) .
\end{aligned}
$$

From the fact that $\left|\eta^{\prime}\right| \leq C \eta^{1 / 2}$ it follows, by using the Cauchy-Schwarz inequality, that

$$
\int_{E_{B}(A)}\left|\eta^{\prime}\left(x_{2}\right) \bar{u} \partial_{2} u\right| d x \leq \frac{1}{2} \int_{E_{B}(A)} \eta\left(x_{2}\right)\left|\partial_{2} u\right|^{2} d x+\frac{C^{2}}{2} \int_{E_{B}(A)}|u|^{2} d x,
$$

so that

$$
\begin{aligned}
\int_{E_{B / 2}(A)}|\nabla u|^{2} d x & \leq \int_{E_{B}(A)} \eta\left(x_{2}\right)|\nabla u|^{2} d x \\
& \leq\left(2\|k\|_{\infty}^{2}+C^{2}\right) \int_{E_{B}(A)}|u|^{2} d x+2 R_{1}(A) .
\end{aligned}
$$

Now, for any $h>0$,

$$
u\left(\left(x_{1}, h\right)\right)-u\left(\left(x_{1}, 0\right)\right)=\int_{0}^{h} \partial_{2} u(x) d x_{2}, \quad x_{1} \in \mathbf{R},
$$

so that, using the Cauchy-Schwarz inequality,

$$
\left|u\left(\left(x_{1}, 0\right)\right)\right|^{2} \leq 2\left|u\left(\left(x_{1}, h\right)\right)\right|^{2}+2 h \int_{0}^{h}\left|\partial_{2} u(x)\right|^{2} d x_{2}, \quad x_{1} \in \mathbf{R} .
$$

From (28) it follows that

$$
\int_{\Gamma(A)}|u|^{2} d s \leq 2 \int_{\Gamma_{h}(A)}|u|^{2} d s+2 h \int_{E_{h}(A)}\left|\partial_{2} u\right|^{2} d x
$$


for any $h>0$ and therefore that

$$
B \int_{\Gamma(A)}|u|^{2} d s \leq 4 \int_{E_{B / 2}(A)}|u|^{2} d x+\frac{B^{2}}{2} \int_{E_{B / 2}(A)}\left|\partial_{2} u\right|^{2} d x .
$$

Thus, assuming that (A3) holds, the required inequality (24), with $C_{1}=C_{2}=C_{5}=0$, follows from (30), (26), and (25).

Suppose now that (A4) and (A5) hold. Multiplying (1) by $2 x_{2} \partial_{2} \bar{u}+\bar{u}$, integrating over $E_{a}(A)$, and taking the real part, we obtain

$$
\begin{aligned}
2 \int_{E_{a}(A)}\left|\partial_{2} u\right|^{2} d x= & \Re \int_{E_{a}(A)}\left[2 \nabla \cdot\left(x_{2} \partial_{2} \bar{u} \nabla u\right)-\partial_{2}\left(x_{2}|\nabla u|^{2}\right)+\nabla \cdot(\bar{u} \nabla u)\right] d x \\
& +\int_{E_{a}(A)} \Re\left(k^{2}\right) \partial_{2}\left(x_{2}|u|^{2}\right) d x+2 \int_{E_{a}(A)} x_{2} \Im\left(k^{2}\right) \Im\left\{\bar{u} \partial_{2} u\right\} d x \\
= & a \int_{\Gamma_{a}(A)}\left(\left|\partial_{2} u\right|^{2}-\left|\partial_{1} u\right|^{2}\right) d s+L_{A}+R_{2}(A) \\
& +\int_{E_{a}(A)} \Re\left(k^{2}\right) \partial_{2}\left(x_{2}|u|^{2}\right) d x+2 \int_{E_{a}(A)} x_{2} \Im\left(k^{2}\right) \Im\left\{\bar{u} \partial_{2} u\right\} d x .
\end{aligned}
$$

Now if $\Re\left(k^{2}\right) \in C^{1}\left(\overline{E_{a}(A)}\right)$ then from (A4) we have that $\partial_{2}\left(\Re\left(k^{2}\right)\right) \geq 0$ and, integrating by parts, we obtain that

$$
\int_{E_{a}(A)} \Re\left(k^{2}\right) \partial_{2}\left(x_{2}|u|^{2}\right) d x \leq k_{+}^{2} a \int_{\Gamma_{a}(A)}|u|^{2} d s=\int_{E_{a}(A)} k_{+}^{2} \partial_{2}\left(x_{2}|u|^{2}\right) d x .
$$

Thus

$$
G_{A}:=\int_{E_{a}(A)}\left[k_{+}^{2}-\Re\left(k^{2}\right)\right] \partial_{2}\left(x_{2}|u|^{2}\right) d x \geq 0 .
$$

In the general case when $k \in L_{\infty}(U)$, let $\phi(x)=\Re\left(k^{2}(x)\right), \psi(x)=x_{2}|u(x)|^{2}$ and, for $h \in \mathbf{R}$, let $\phi_{h}(x)=\phi\left(x+h e_{2}\right), \psi_{h}(x)=\psi\left(x+h e_{2}\right)$. Then, since $\phi\left(\psi_{h}-\psi\right)+\phi_{h}\left(\psi_{h}-\right.$ $\psi)=2\left(\phi_{h} \psi_{h}-\phi \psi\right)-\left(\phi_{h}-\phi\right)\left(\psi+\psi_{h}\right)$, we have that

$$
\begin{aligned}
& \int_{E_{a}(A)} \phi\left(\psi_{h}-\psi\right) d x+\int_{E_{a+h}(A) \backslash E_{h}(A)} \phi\left(\psi-\psi_{-h}\right) d x \\
= & 2 \int_{E_{a+h}(A) \backslash E_{a}(A)} \phi \psi d x-2 \int_{E_{h}(A)} \phi \psi d x-\int_{E_{a}(A)}\left(\phi_{h}-\phi\right)\left(\psi+\psi_{h}\right) d x \\
\leq & 2 k_{+}^{2} \int_{E_{a+h}(A) \backslash E_{a}(A)} \psi d x+2\|k\|_{\infty}^{2} \int_{E_{h}(A)} \psi d x
\end{aligned}
$$

on using (A2) and (A4). Since $\psi \in C^{1}(\bar{U})$ and $\psi=0$ on $\Gamma$, dividing (34) by $2 h$ and taking the limit $h \rightarrow 0$ we obtain that (32) and (33) hold in the general case.

It follows from (31) and (32) that

$$
\text { (35) } 2 \int_{E_{a}(A)}\left|\partial_{2} u\right|^{2} d x+G_{A}=a I_{A}+L_{A}+R_{2}(A)+2 \int_{E_{a}(A)} x_{2} \Im\left(k^{2}\right) \Im\left\{\bar{u} \partial_{2} u\right\} d x .
$$

Since $0 \leq \Im\left(k^{2}\right) \leq\|k\|_{\infty}^{2}$, the Cauchy-Schwarz inequality yields that

$$
2 \int_{E_{a}(A)} x_{2} \Im\left(k^{2}\right) \Im\left\{\bar{u} \partial_{2} u\right\} d x \leq \int_{E_{a}(A)}\left|\partial_{2} u\right|^{2} d x+a^{2}\|k\|_{\infty}^{2} \int_{E_{a}(A)} \Im\left(k^{2}\right)|u|^{2} d x
$$


Thus, it follows from (35), (36), and (25) that

$$
\int_{E_{a}(A)}\left|\partial_{2} u\right|^{2} d x+G_{A} \leq a I_{A}+L_{A}-a^{2}\|k\|_{\infty}^{2} J_{A}+R_{2}(A)+a^{2}\|k\|_{\infty}^{2} R_{1}(A)=: F_{A} .
$$

Now, from (37) and the fact that $G_{A} \geq 0$, it is seen that

$$
\int_{E_{a}(A)}\left|\partial_{2} u\right|^{2} d x \leq F_{A}
$$

On the other hand, since $2\left|x_{2} \Re\left(\bar{u} \partial_{2} u\right)\right| \leq|u|^{2} / 2+2 a^{2}\left|\partial_{2} u\right|^{2}$ for $x \in E_{a}$, we have

$$
\partial_{2}\left(x_{2}|u|^{2}\right)=|u|^{2}+2 x_{2} \Re\left(\bar{u} \partial_{2} u\right) \geq|u|^{2} / 2-2 a^{2}\left|\partial_{2} u\right|^{2}
$$

for $x \in E_{a}$, so that, on noting that $k_{+}^{2} \geq \Re\left(k^{2}\right)$ by (A4),

$$
G_{A} \geq \frac{1}{2} \int_{E_{a}(A)}\left[k_{+}^{2}-\Re\left(k^{2}\right)\right]|u|^{2} d x-4 a^{2}\|k\|_{\infty}^{2} \int_{E_{a}(A)}\left|\partial_{2} u\right|^{2} d x
$$

This, together with (37), implies that

$$
\begin{aligned}
\int_{E_{a}(A)}\left[k_{+}^{2}-\Re\left(k^{2}\right)\right]|u|^{2} d x & \leq 2 F_{A}+8 a^{2}\|k\|_{\infty}^{2} \int_{E_{a}(A)}\left|\partial_{2} u\right|^{2} d x \\
& \leq 2\left(1+4 a^{2}\|k\|_{\infty}^{2}\right) F_{A} .
\end{aligned}
$$

We now make use of (38) and (39) to derive the required inequality (24). First, using (28) we obtain that, for any $0<h \leq a$,

$$
\begin{array}{r}
\int_{E_{h}(A)}\left[k_{+}^{2}-\Re\left(k^{2}\right)\right]\left|u\left(x_{1}, 0\right)\right|^{2} d x \leq \int_{E_{a}(A)}\left[k_{+}^{2}-\Re\left(k^{2}\right)\right]\left|u\left(x_{1}, 0\right)\right|^{2} d x \\
\leq 2 \int_{E_{a}(A)}\left[k_{+}^{2}-\Re\left(k^{2}\right)\right]|u|^{2} d x+4 a^{2}|| k \|_{\infty}^{2} \int_{E_{a}(A)}\left|\partial_{2} u\right|^{2} d x .
\end{array}
$$

Taking $h=d$ in (40) and using (A5) yield that

$$
d \lambda_{2} \int_{\Gamma(A)}|u|^{2} d s \leq 2 \int_{E_{a}(A)}\left[k_{+}^{2}-\Re\left(k^{2}\right)\right]|u|^{2} d x+4 a^{2}\|k\|_{\infty}^{2} \int_{E_{a}(A)}\left|\partial_{2} u\right|^{2} d x
$$

Next, using (27) we get (cf. (29))

$$
\int_{\Gamma_{h}(A)}|u|^{2} d s \leq 2 \int_{\Gamma(A)}|u|^{2} d s+2 h \int_{E_{h}(A)}\left|\partial_{2} u\right|^{2} d x
$$

for any $h>0$, and therefore

$$
\int_{E_{a}(A)}|u|^{2} d x \leq 2 a \int_{\Gamma(A)}|u|^{2} d s+a^{2} \int_{E_{a}(A)}\left|\partial_{2} u\right|^{2} d x .
$$

Thus, utilizing (38) and (39) together with (41) and (42), the required result (24) follows. The proof is complete. 
5. Uniqueness of solution. In this section we establish the following uniqueness theorem for problem $(\mathrm{P})$.

THEOREM 5.1. If (A3) holds or both (A4) and (A5) hold, then problem (P) has at most one solution.

We prove this theorem by showing that the homogeneous version of problem $(\mathrm{P})$ has only the trivial solution. Since guided waves are solutions of the homogeneous problem (see the definition and Theorem B.2 in Appendix B), we have immediately the following corollary.

COROLlaRY 5.2. If (A3) holds or both (A4) and (A5) hold, then there are no guided wave solutions to the homogeneous problem.

In the proof of Theorem 5.1 we utilize the following two lemmas, of which the first is a consequence of the UPRC and was proved as Lemma 6.1 in [6] and the second is a special case of Lemma $\mathrm{A}$ in [7].

Lemma 5.3. If $\phi \in L_{2}\left(\Gamma_{h}\right) \cap L_{\infty}\left(\Gamma_{h}\right)$ and $v$ is defined by (3), then the restrictions of $v, \partial_{1} v$, and $\partial_{2} v$ to $\Gamma_{a}$ are in $L_{2}\left(\Gamma_{a}\right) \cap B C\left(\Gamma_{a}\right)$ for $a>h$ and

$$
\begin{aligned}
& \Im \int_{\Gamma_{a}} \bar{v} \partial_{2} v d s \geq 0, \quad \Re \int_{\Gamma_{a}} \bar{v} \partial_{2} v d s \leq 0, \\
& \int_{\Gamma_{a}}\left[\left|\partial_{2} v\right|^{2}-\left|\partial_{1} v\right|^{2}+k_{+}^{2}|v|^{2}\right] d s \leq 2 k_{+} \Im \int_{\Gamma_{a}} \bar{v} \partial_{2} v d s .
\end{aligned}
$$

Lemma 5.4. Suppose that $F \in L_{\infty}(\mathbf{R})$ and that, for some nonnegative constants $c, \epsilon$, and $A_{0}$,

$$
\int_{-A}^{A}|F|^{2} \leq c \int_{\mathbf{R} \backslash[-A, A]} G_{A}^{2}+c \int_{-A}^{A}\left(G_{\infty}-G_{A}\right) G_{\infty}+\epsilon, \quad A>A_{0},
$$

where, for $A_{0}<A \leq+\infty$,

$$
G_{A}(s)=\int_{-A}^{A}(1+|s-t|)^{-3 / 2}|F(t)| d t, \quad s \in \mathbf{R} .
$$

Then $F \in L_{2}(\mathbf{R})$ and

$$
\int_{-\infty}^{+\infty}|F|^{2} \leq \epsilon
$$

Proof (of Theorem 5.1). Suppose that $u_{1}$ and $u_{2}$ are solutions of problem (P). Then, by Remark 2.2, $u=u_{1}-u_{2} \in C^{1}(\bar{U})$ and satisfies (1)-(2), the bound (7), and the UPRC. Also, by Theorem 3.3,

(45) $u(x)=\int_{E_{B}} u(y)\left[k^{2}(y)-k_{+}^{2}\right] G(x, y) d y-i k_{+} \int_{\Gamma} u(y) G(x, y) d s(y), \quad x \in \bar{U}$,

and, by Theorem 4.1, for some constants $a>B$ and $C_{j} \geq 0, j=1,2,3,4,5$,

$$
K_{A} \leq C_{1} I_{A}+C_{2} L_{A}-C_{3} J_{A}+C_{4} R_{1}(A)+C_{5} R_{2}(A),
$$

where $J_{A}, I_{A}, L_{A}$, and $K_{A}$ are given by (22) and (23). Clearly, for $j=1,2$,

$$
R_{j}(A)=O(1) \text { as } A \rightarrow \infty
$$


and, by (25),

$$
J_{A}=R_{1}(A)-\int_{E_{B}(A)} \Im\left(k^{2}\right)|u|^{2} d x \leq R_{1}(A) .
$$

Now to make use of Lemma 5.3 and the bound (46) we define

(49) $v(x)=\int_{E_{B}(A)} u(y)\left[k^{2}(y)-k_{+}^{2}\right] G(x, y) d y-i k_{+} \int_{\Gamma(A)} u(y) G(x, y) d s(y), \quad x \in \bar{U}$.

Then, by (13), (14), and Lemmas 3.1 and $3.2,\left.v\right|_{\Gamma_{B}} \in L_{2}\left(\Gamma_{B}\right) \cap B C\left(\Gamma_{B}\right)$; and by Remark 2.3, Lemmas 3.1 and 3.2, and the equivalence of (i) and (iv) in Theorem 2.3, $v$ satisfies (3) with $h=B$ and $\phi=\left.v\right|_{\Gamma_{B}}$. Set

$$
\begin{array}{cc}
J_{A}^{\prime}=\Im \int_{\Gamma_{a}(A)} \bar{v} \partial_{2} v d s, \quad J_{A}^{\prime \prime}=\Im \int_{\Gamma_{a}} \bar{v} \partial_{2} v d s, \\
I_{A}^{\prime}=\int_{\Gamma_{a}(A)}\left\{\left|\partial_{2} v\right|^{2}-\left|\partial_{1} v\right|^{2}+k_{+}^{2}|v|^{2}\right\} d s, & I_{A}^{\prime \prime}=\int_{\Gamma_{a}}\left\{\left|\partial_{2} v\right|^{2}-\left|\partial_{1} v\right|^{2}+k_{+}^{2}|v|^{2}\right\} d s, \\
L_{A}^{\prime}=\Re \int_{\Gamma_{a}(A)} \bar{v} \partial_{2} v d s, & L_{A}^{\prime \prime}=\Re \int_{\Gamma_{a}} \bar{v} \partial_{2} v d s .
\end{array}
$$

Then, by Lemma 5.3,

$$
J_{A}^{\prime \prime} \geq 0, \quad L_{A}^{\prime \prime} \leq 0, \quad I_{A}^{\prime \prime} \leq 2 k_{+} J_{A}^{\prime \prime},
$$

so that, by (46) and (48),

$$
\begin{aligned}
K_{A} \leq & C_{1}\left(I_{A}-I_{A}^{\prime \prime}\right)+\left(2 C_{1} k_{+}+C_{3}\right)\left(J_{A}^{\prime \prime}-J_{A}\right) \\
& +C_{2}\left(L_{A}-L_{A}^{\prime \prime}\right)+\left(2 C_{1} k_{+}+C_{4}\right) R_{1}(A)+C_{5} R_{2}(A) .
\end{aligned}
$$

Now note that

$$
K_{A}=\int_{-A}^{A}\left|w\left(x_{1}\right)\right|^{2} d x_{1}
$$

where

$$
w\left(x_{1}\right)=\left\{\int_{0}^{B}|u(x)|^{2} d x_{2}+\left|u\left(x_{1}, 0\right)\right|^{2}\right\}^{1 / 2}, \quad x_{1} \in \mathbf{R},
$$

and that, by (15) and the Cauchy-Schwarz inequality,

$$
\begin{aligned}
|v(x)|,|\nabla v(x)| & \leq C_{k}(B+1)^{1 / 2} W_{A}\left(x_{1}\right), \quad x \in \Gamma_{a}, \\
|u(x)-v(x)|,|\nabla u(x)-\nabla v(x)| & \leq C_{k}(B+1)^{1 / 2}\left(W_{\infty}\left(x_{1}\right)-W_{A}\left(x_{1}\right)\right), \quad x \in \Gamma_{a},
\end{aligned}
$$

where $C_{k}=C_{a} \max \left(k_{+},\left\|k_{+}^{2}-k^{2}\right\|_{\infty}\right)$ and, for $0 \leq A \leq+\infty$,

$$
W_{A}\left(x_{1}\right)=\int_{-A}^{A}\left(1+\left|x_{1}-y_{1}\right|\right)^{-3 / 2} w\left(y_{1}\right) d y_{1}, \quad x_{1} \in \mathbf{R} .
$$


It follows that

$$
\left|I_{A}^{\prime}-I_{A}^{\prime \prime}\right|,\left|J_{A}^{\prime}-J_{A}^{\prime \prime}\right|,\left|L_{A}^{\prime}-L_{A}^{\prime \prime}\right| \leq C \int_{\mathbf{R} \backslash[-A, A]}\left(W_{A}\left(x_{1}\right)\right)^{2} d x_{1},
$$

where $C=C_{k}^{2}(B+1)\left(2+k_{+}^{2}\right)$, and that

$$
\left|I_{A}-I_{A}^{\prime}\right|,\left|J_{A}-J_{A}^{\prime}\right|,\left|L_{A}-L_{A}^{\prime}\right| \leq 2 C \int_{-A}^{A}\left(W_{\infty}\left(x_{1}\right)-W_{A}\left(x_{1}\right)\right) W_{\infty}\left(x_{1}\right) d x_{1},
$$

so that, for some constant $c>0$ and all $A>0$,

$$
K_{A} \leq c\left\{\int_{\mathbf{R} \backslash[-A, A]} W_{A}^{2}+\int_{-A}^{A}\left(W_{\infty}-W_{A}\right) W_{\infty}+\left|R_{1}(A)\right|+\left|R_{2}(A)\right|\right\} .
$$

Applying Lemma 5.4 to (51) we obtain that $w \in L_{2}(\mathbf{R})$ (i.e. $u \in L_{2}\left(E_{B}\right) \cap L_{2}(\Gamma)$ ) and, for all $A_{0}>0$,

$$
\int_{E_{B}}|u|^{2} d x+\int_{\Gamma}|u|^{2} d s=\int_{-\infty}^{+\infty}|w|^{2} \leq c \sup _{A>A_{0}}\left(\left|R_{1}(A)\right|+\left|R_{2}(A)\right|\right) .
$$

Since $u \in L_{2}\left(E_{B}\right) \cap L_{2}(\Gamma)$, it follows from (45), the bounds (13) and (14), and applications of Young's theorem, that $u \in L_{2}\left(E_{a}\right)$, for every $a>0$. Since also $\nabla u \in B C\left(E_{a}\right)$ so that $u$ is uniformly continuous in $\overline{E_{a}}$, it follows that $u(x) \rightarrow 0$ as $x_{1} \rightarrow \infty$, uniformly in $x_{2}$ for $0 \leq x_{2} \leq a$, for every $a>0$. Noting also Lemma 2.4, it follows that $R_{j}(A) \rightarrow 0$ as $A \rightarrow \infty, j=1,2$, and thus, from (52), that $u=0$ in $E_{B}$ and on $\Gamma$ and hence, from (45), that $u \equiv 0$ in $\bar{U}$. The proof is complete.

6. Existence of solution. In this section we apply Theorems A.1 and A.2 in Appendix A to show the existence of a solution to problem (P). First, we note that (see Remark 3.1) problem (P) and (17) are equivalent. Let $N=2, \Omega_{1}=E_{B} \subset \mathbf{R}^{2}$, $\Omega_{2}=\mathbf{R}, \omega_{1}=\left\{x \in \Omega_{1} \mid 0 \leq x_{1}<B, 0<x_{2}<B\right\}, \omega_{2}=[0, B), a_{1}^{(1)}=(B, 0)$, and $a_{1}^{(2)}=B$. Define $\tilde{y}:=y$ for $y \in \Omega_{1} \subset \mathbf{R}^{2}$ and $\tilde{y}:=(y, 0)$ for $y \in \Omega_{2}=\mathbf{R}$, and let $k_{i j}(x, y)=G(\tilde{x}, \tilde{y})$ for all $x \in \Omega_{i}, y \in \Omega_{j}, \tilde{x} \neq \tilde{y}, i, j=1,2$. Then conditions (C.1) and (C.2) in Appendix A are satisfied with these choices of $k_{i j}$ and $\Omega_{j}(i, j=1,2)$. Set $w_{1}(y)=k^{2}(y)-k_{+}^{2}$ for $y \in \Omega_{1}$ and $w_{2}(y)=-i k_{+}$for $y \in \Omega_{2}$. Then the integral equation (17) is equivalent to the equation

$$
u(x)=u^{\mathrm{i}}(x)+u^{\mathrm{r}}(x)+\int_{\Omega_{1}} \psi_{1}(y) w_{1}(y) G(x, y) d \mu_{2}(y)+\int_{\Omega_{2}} \psi_{2}(y) w_{2}(y) G(x, \tilde{y}) d \mu_{1}(y)
$$

for $x \in \bar{U}$, where $\psi_{1}(y)=u(y)$ for $y \in \bar{\Omega}_{1}, \psi_{2}(y)=u(\tilde{y})$ for $y \in \Omega_{2}$, and $d \mu_{j}$ is $j$-dimensional Lebesgue measure.

With $X$ and $Y$ the product spaces as defined in Appendix A, let us now consider the system of integral equations

$$
\left(I-K_{w}\right) \psi=\phi, \quad \psi=\left(\psi_{1}, \psi_{2}\right)^{t}, \quad \phi=\left(\phi_{1}, \phi_{2}\right)^{t} \in Y, \quad w=\left(w_{1}, w_{2}\right)^{t} \in X,
$$

where $\phi_{j}(y)=u^{\mathrm{i}}(\tilde{y})+u^{\mathrm{r}}(\tilde{y})$ for $y \in \bar{\Omega}_{j}(j=1,2)$, and $I$ and $K_{w}$ are the matrix operators defined by (A.4) and (A.5). Then it is clear that (54) is just a restriction of (53) to $\overline{E_{B}}$ and $\Gamma$, so that if we can solve (54) for $\psi$, then $u$, defined by (53), is a solution to problem $(\mathrm{P})$. Therefore, in order to prove the existence of a solution to 
problem $(\mathrm{P})$, it is enough to show that the system of integral equations (54) has a solution.

TheOREM 6.1. Assume that (A3) holds or that both (A4) and (A5) hold. Then $\left(I-K_{w}\right)^{-1} \in B(Y)$ so that the system of integral equations (54) has a unique solution $\psi \in Y$. Furthermore, for any $M>0$, there is a constant $C>0$ depending only on $M$, $k_{+}, B$, and $\lambda_{1}$ in the case that (A3) is satisfied or on $M, k_{+}, B, \lambda_{2}$, and $d$ in the case that (A4) and (A5) are satisfied, such that, provided $\|k\|_{\infty} \leq M$, it holds that $\left\|\left(I-K_{w}\right)^{-1}\right\| \leq C$, so that $\|\psi\| \leq C\|\phi\|$.

Proof. Theorem 6.1 is proved by means of Theorems A.1 and A.2 in Appendix A. To this end, suppose without loss of generality that $M>\lambda_{1}$, and set

$$
Q=Q_{3}:=\left\{\mu \in L_{\infty}\left(\Omega_{1}\right) \mid \Im \mu \geq \lambda_{1},\|\mu\|_{\infty} \leq M^{2}\right\}
$$

in the case that (A3) is satisfied. In the case that (A4) and (A5) are satisfied, suppose without loss of generality that $M>k_{+}$, and set

$$
\begin{aligned}
Q=Q_{4}:=\{ & \mu \in L_{\infty}(U) \mid \Im \mu \geq 0, \mu(x)=k_{+}^{2}, x \in U_{B},\|\mu\|_{\infty} \leq M^{2}, \operatorname{ess}_{\sup _{x \in E_{d}} \Re(\mu(x))} \\
& \left.\leq k_{+}^{2}-\lambda_{2}, \operatorname{ess}_{\inf _{x \in U}} \Re\left[\mu\left(x+e_{2} h\right)-\mu(x)\right] \geq 0, h>0\right\} .
\end{aligned}
$$

With $X_{j}=L_{\infty}\left(\Omega_{j}\right), j=1,2$, set

$$
\begin{aligned}
W_{1} & =\left\{z_{1} \in X_{1}\left|z_{1}=\mu\right|_{\Omega_{1}}-k_{+}^{2}, \mu \in Q\right\}, \\
W_{2} & =\left\{z_{2} \in X_{2} \mid z_{2}(x)=-i k_{+}, x \in \Omega_{2}\right\}, \\
W & =\left\{z=\left(z_{1}, z_{2}\right)^{t} \in X \mid z_{1} \in W_{1}, z_{2} \in W_{2}\right\} .
\end{aligned}
$$

Then $T_{a} W=W$ for $a \in \tau=\left\{\left(a_{1}^{(1)}, a_{1}^{(2)}\right)^{t}\right\}$. Also, it follows easily from Theorems 3.4 and 5.1 that $I-K_{z}$ is injective for all $z \in W$.

Next, we show that $W$ is weak* sequentially compact. From Appendix A, it is sufficient to show that $W_{j} \subset X_{j}$ is weak* sequentially compact for $j=1,2$. Clearly $W_{2}=\left\{-i k_{+}\right\}$is weak* sequentially compact. As $\left\{w \in \mathbf{C}|| w \mid \leq M^{2}\right.$, ऽw $\left.\geq \lambda_{1}\right\}$ is compact and convex, it follows from Lemma 2.13 in [8] in the case $Q=Q_{3}$ that $W_{1}$ is weak* sequentially compact. In the case $Q=Q_{4}$, let $z_{1}^{(j)}=\left.\mu^{(j)}\right|_{\Omega_{1}}-k_{+}^{2} \in W_{1}$, $j=1,2, \ldots$. Since $\left(\mu^{(j)}\right) \subset Q$, which is bounded, it follows from the Alaoglu theorem $[17$, p. 60$]$ that there is an element $\mu \in L_{\infty}(U)$ and a subsequence of $\left(\mu^{(j)}\right)$, denoted simply by itself, such that $\left(\mu^{(j)}\right)$ converges weak* to $\mu$ in $L_{\infty}(U)$ and $\|\mu\|_{\infty} \leq M^{2}$. Thus, for all $\xi \in L_{1}(U)$,

$$
\int_{U} \mu^{(j)} \xi d x \rightarrow \int_{U} \mu \xi d x
$$

as $j \rightarrow \infty$, and, in particular, (55) holds if $\xi$ is the characteristic function of any bounded measurable subset of $U$. This and that $\mu^{(j)} \in Q, j=1,2, \ldots$ implies that $\Im \mu \geq 0$ in $U, \mu(x)=k_{+}^{2}$ for $x \in U_{B}$, ess $\sup _{x \in E_{d}} \Re(\mu(x)) \leq k_{+}^{2}-\lambda_{2}$, and $\operatorname{ess}_{\inf _{x \in U}} \Re\left[\mu\left(x+e_{2} h\right)-\mu(x)\right] \geq 0$ for all $h \geq 0$. Hence, $\mu \in Q, z_{1}=\left.\mu\right|_{\Omega_{1}}-k_{+}^{2} \in W_{1}$ and $\left(z_{1}^{(j)}\right)$ converges weak $*$ to $z_{1}$ in $X_{1}$, that is, $W_{1}$ is weak* sequentially compact.

Finally, let $z=\left(z_{1}, z_{2}\right)^{t} \in W$ with $z_{1}=\left.\mu\right|_{\Omega_{1}}-k_{+}^{2} \in W_{1}$ and $z_{2}=-i k_{+} \in W_{2}$. For $j=1,2, \ldots$ set

$$
\mu^{(j)}(x)= \begin{cases}\tilde{\mu}(x) & \text { for }\left|x_{1}\right|>j \\ \mu(x) & \text { for }\left|x_{1}\right| \leq j\end{cases}
$$


where $\tilde{\mu} \equiv i \lambda_{1}$ in the case that (A3) is satisfied, $\tilde{\mu}(x)=k_{+}^{2}-\lambda_{2}, x \in E_{B},=k_{+}^{2}, x \in U_{B}$ in the case that (A4) and (A5) are satisfied. Then $\tilde{\mu}, \mu^{(j)} \in Q, z_{1}^{(j)}=\left.\mu^{(j)}\right|_{\Omega_{1}}-k_{+}^{2} \in$ $W_{1}$, and $z_{1}^{(j)}(x)=z_{1}^{*},\left|x_{1}\right|>j$, where $z_{1}^{*}=\mu^{*}-k_{+}^{2}$ and $\mu^{*}=i \lambda_{1}$ in the case that (A3) is satisfied and $\mu^{*}=k_{+}^{2}-\lambda_{2}$ in the case that (A4) and (A5) are satisfied. For any $\xi \in L_{1}\left(\Omega_{1}\right)$ we have

$$
\int_{\Omega_{1}}\left(z_{1}^{(j)}-z_{1}\right) \xi d x=\int_{E_{B} \backslash E_{B}(j)}\left(\mu-\mu^{*}\right) \xi d x \leq 2 M^{2} \int_{E_{B} \backslash E_{B}(j)}|\xi| d x \rightarrow 0
$$

as $j \rightarrow \infty$, that is, $\left(z_{1}^{(j)}\right)$ converges weak* to $z_{1}$ in $X_{1}$. Now for $j=1,2, \ldots$ set $z^{(j)}=\left(z_{1}^{(j)}, z_{2}\right)^{t}$. Then $z^{(j)} \in W$ and $\left(z^{(j)}\right)$ converges weak* to $z$ in $X$. Set $z^{*}=$ $\left(z_{1}^{*}, z_{2}\right)^{t}$. Since $z^{*} \in W$ so that $I-K_{z^{*}}$ is injective, it follows from Theorem A.2 in Appendix A that $I-K_{z^{(j)}}$ injective implies $I-K_{z^{(j)}}$ surjective, for $j=1,2, \ldots$. Thus all the assumptions in Theorem A.1 have been verified, and Theorem 6.1 follows from Theorem A.1. The proof is complete.

TheOREM 6.2. Assume that (A3) holds or both (A4) and (A5) hold. Then problem $(\mathrm{P})$ has exactly one solution. Further, for any $M>0$, there exists a constant $C>0$ depending only on $M, k_{+}, B$, and $\lambda_{1}$ in the case that (A3) is satisfied or on $M, k_{+}, B, \lambda_{2}$, and $d$ in the case that (A4) and (A5) are satisfied, such that, provided $\|k\|_{\infty} \leq M$

$$
|u(x)| \leq C\left(1+x_{2}\right)^{1 / 2}, \quad x \in \bar{U} .
$$

Proof. The existence of a unique solution to problem (P) follows from Theorems $3.3,3.4$, and 6.1. To derive the estimate (56) we note from the equivalence of (i) and (iv) in Lemma 2.3 that

$$
u^{\mathrm{s}}(x)=2 \int_{\Gamma_{B}} \frac{\partial \Phi(x, y)}{\partial y_{2}} u^{\mathrm{s}}(y) d s(y), \quad x \in U_{B} .
$$

It follows from (4) and (57) (see [4]) that

$$
\left|u^{\mathrm{s}}(x)\right| \leq C\left(1+\left(x_{2}-B\right)\right)^{1 / 2}\left\|u_{B}^{\mathrm{s}}\right\|_{\infty}, \quad x \in U_{B},
$$

for some constant $C>0$ dependent only on $k_{+}$, which together with Theorem 6.1 implies the estimate (56). The proof is complete.

We remark that in [6] it is shown for the Dirichlet boundary condition case that, for a particular choice of $k$, the growth rate in (56) for the solution can be achieved, so that the exponent $1 / 2$ in the bound (56) is the best possible for the general case.

\section{Appendix A. Some solvability results for integral equations on un- bounded domains.}

In this appendix we summarize general results on the solvability of a class of systems of second kind integral equations on unbounded domains established in [9] and used in section 6 to show existence of solution for the scattering problem.

Let us consider the system of second kind integral equations

$$
\psi_{i}(x)=\phi_{i}(x)+\sum_{j=1}^{N} \int_{\Omega_{j}} k_{i j}(x, y) z_{j}(y) \psi_{j}(y) d \mu_{j}(y), \quad x \in \bar{\Omega}_{i}, \quad i=1, \ldots, N,
$$

where $\Omega_{j}$ is an open subset of $\mathbf{R}^{n_{j}}\left(n_{j} \geq 1\right), d \mu_{j}$ is $n_{j}$-dimensional Lebesgue measure, the functions $k_{i j}: \bar{\Omega}_{i} \times \Omega_{j} \rightarrow \mathbf{C}, \phi_{j} \in Y_{j}:=B C\left(\bar{\Omega}_{j}\right)$, and $z_{j} \in X_{j}:=L_{\infty}\left(\Omega_{j}\right)$ are 
known, and $\psi_{j} \in Y_{j}$ is to be determined $(i, j=1, \ldots, N)$. As usual, for $a \in \mathbf{R}^{n}$ and $V \subset \mathbf{R}^{n}$, denote by $V+a$ the translate of the set $V$ by the vector $a$. We assume throughout this appendix that $k_{i j}(x, \cdot) \in L_{1}\left(\Omega_{j}\right)$ for every $x \in \bar{\Omega}_{i}(i, j=1, \ldots, N)$, so that the integrals in (A.1) are well defined. We also assume that the following conditions on $k_{i j}$ and $\Omega_{j}$ hold.

(C.1) $\sup _{x \in \bar{\Omega}_{i}} \int_{\Omega_{j}}\left|k_{i j}(x, y)\right| d \mu_{j}(y)<\infty$; and, for all $x \in \bar{\Omega}_{i}$,

$$
\int_{\Omega_{j}}\left|k_{i j}(x, y)-k_{i j}\left(x^{\prime}, y\right)\right| d \mu_{j}(y) \rightarrow 0
$$

as $x^{\prime} \rightarrow x$ with $x^{\prime} \in \bar{\Omega}_{i}(i, j=1, \ldots, N)$.

(C.2) For some $m \leq \min _{j} n_{j}$ and $i=1, \ldots, N$, there exists $a_{j}^{(i)} \in \mathbf{R}^{n_{i}}, j=$ $1, \ldots, m$, and a bounded set $\omega_{i} \subset \Omega_{i}$ such that

(i) $\Omega_{i}=\bigcup_{P \in \mathbf{Z}^{m}} \omega_{i}^{(P)}$, where $\omega_{i}^{(P)}:=\omega_{i}+\sum_{j=1}^{m} a_{j}^{(i)} p_{j}$ for $P=\left(p_{1}, \ldots, p_{m}\right) \in \mathbf{Z}^{m}$;

(ii) $\omega_{i}^{(Q)} \cap \omega_{i}^{(P)}=\emptyset$ for $Q, P \in \mathbf{Z}^{m}, Q \neq P$;

(iii) $k_{i j}\left(x+a_{l}^{(i)}, y+a_{l}^{(j)}\right)=k_{i j}(x, y), x \in \bar{\Omega}_{i}, y \in \bar{\Omega}_{j}, i, j=1, \ldots, N, l=1, \ldots, m$.

Define the integral operator $K_{i j}: X_{j} \rightarrow Y_{i}$ by

$$
K_{i j} \psi(x)=\int_{\Omega_{j}} k_{i j}(x, y) \psi(y) d \mu_{j}(y), \quad x \in \bar{\Omega}_{i}, \quad i, j=1, \ldots, N .
$$

Then (A.1) can be abbreviated as

$$
\psi_{i}=\phi_{i}+\sum_{j=1}^{N} K_{i j}\left(z_{j} \psi_{j}\right), \quad i=1, \ldots, N .
$$

Let $Y:=\prod_{j=1}^{N} Y_{j}$ and $X:=\prod_{j=1}^{N} X_{j}$ be the product spaces. For $\phi \in X_{j}$, we denote

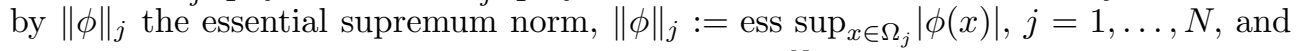
by $\|\phi\|$ the product norm on $X$, defined by $\|\phi\|=\sum_{j=1}^{N}\left\|\phi_{j}\right\|_{j}, \phi=\left(\phi_{1}, \ldots, \phi_{N}\right)^{t} \in X$, where $(\cdot, \ldots, \cdot)^{t}$ denotes the transpose of $(\cdot, \ldots, \cdot)$. Equipped with the product norm $\|\cdot\|$, the product space $X$ is a Banach space, and $Y$ is a closed subspace of $X$. Since $X_{j}$ can be identified with $\left(L_{1}\left(\Omega_{j}\right)\right)^{\prime}$, the dual space of $L_{1}\left(\Omega_{j}\right)(j=1, \ldots, N)$, it follows that $X$ can be identified with $\prod_{j=1}^{N}\left(L_{1}\left(\Omega_{j}\right)\right)^{\prime} \equiv\left(\prod_{j=1}^{N} L_{1}\left(\Omega_{j}\right)\right)^{\prime}$, the dual space of the product space $\prod_{j=1}^{N} L_{1}\left(\Omega_{j}\right)$. Thus, for a sequence $\left(\phi_{n}\right) \subset X$ and $\phi \in X$ with $\phi_{n}=\left(\phi_{n}^{(1)}, \ldots, \phi_{n}^{(N)}\right)^{t}$ and $\phi=\left(\phi^{(1)}, \ldots, \phi^{(N)}\right)^{t},\left(\phi_{n}\right)$ converges to $\phi$ in the weak* topology on $X\left(\phi_{n} \stackrel{w *}{\rightarrow} \phi\right)$ if and only if

$$
\int_{\Omega_{j}} \phi_{n}^{(j)} \psi \rightarrow \int_{\Omega_{j}} \phi^{(j)} \psi \quad \text { for all } \psi \in L_{1}\left(\Omega_{j}\right), \quad j=1, \ldots, N,
$$

i.e. if and only if $\phi_{n}^{(j)} \stackrel{w *}{\rightarrow} \phi^{(j)}$ in $X_{j}, j=1, \ldots, N$.

Now define the matrix operator $K$ on $X$ by

$$
K=\left(\begin{array}{ccc}
K_{11} & \cdots & K_{1 N} \\
& \ddots & \\
K_{N 1} & \cdots & K_{N N}
\end{array}\right) .
$$

Then (A.2) can be further abbreviated in matrix operator notation as

$$
\psi=\phi+K_{z} \psi=\phi+K(\hat{z} \psi)
$$


where, for $z=\left(z_{1}, \ldots, z_{N}\right)^{t} \in X, \hat{z}$ is defined as

$$
\hat{z}=\left(\begin{array}{ccc}
z_{1} & & 0 \\
& \ddots & \\
0 & & z_{N}
\end{array}\right)
$$

and $K_{z}: Y \rightarrow Y$ is defined by

$$
K_{z} \psi=K(\hat{z} \psi), \quad \psi \in Y .
$$

For $l=1, \ldots, m$, define the translation operator $T_{a_{l}^{(j)}}: X_{j} \rightarrow X_{j}$ by

$$
T_{a_{l}^{(j)}} \psi(x)=\psi\left(x-a_{l}^{(j)}\right), \quad x \in \bar{\Omega}_{j} \quad(j=1, \ldots, N),
$$

and, for $a=\left(a_{1}, \ldots, a_{N}\right) \in \tau:=\left\{\left(a_{l}^{(1)}, \ldots, a_{l}^{(N)}\right)^{t} \mid l=1, \ldots, m\right\}$, the matrix operator $T_{a}: X \rightarrow X$ by

$$
T_{a}=\left(\begin{array}{ccc}
T_{a_{1}} & & 0 \\
& \ddots & \\
0 & & T_{a_{N}}
\end{array}\right) .
$$

Then by (C.2) (iii), $T_{a} K=K T_{a}$ for $a \in \tau$. Let $B(Y)$ denote the Banach space of bounded linear operators on $Y$ and $I$ the identity matrix operator on $Y$, given by

$$
I=\left(\begin{array}{ccc}
I_{1} & & 0 \\
& \ddots & \\
0 & & I_{N}
\end{array}\right)
$$

where $I_{j}$ is the identity operator on $Y_{j}(j=1, \ldots, N)$. Then the following results have been proved in [9] on the solvability of the system of integral equations (A.3). These results are straightforward extensions to a system of integral equations of results for a single integral equation established in [8].

Theorem A.1. Suppose that (C.1)-(C.2) are satisfied and that $W \subset X$ is weak* sequentially compact, $T_{a} W=W$ for all $a \in \tau$, and $I-K_{z}$ is injective for all $z \in W$. Then $\left(I-K_{z}\right)^{-1}$ exists as an operator on the range space $\left(I-K_{z}\right) Y$ for all $z \in W$ and

$$
\sup _{z \in W}\left\|\left(I-K_{z}\right)^{-1}\right\|<\infty
$$

If also, for every $z \in W$, there exists a sequence $\left(z_{j}\right) \subset W$ such that $z_{j}$ converges weak* to $z$ and

$$
\text { for all } j, \quad I-K_{z_{j}} \quad \text { injective } \Longrightarrow I-K_{z_{j}} \quad \text { surjective, }
$$

then also $I-K_{z}$ is surjective for each $z \in W$ so that $\left(I-K_{z}\right)^{-1} \in B(Y)$.

Theorem A.2. If (C.1)-(C.2) are satisfied, $z=\left(z_{1}, \ldots, z_{N}\right)^{t} \in X, \lambda=\left(\lambda_{1}, \ldots, \lambda_{N}\right)^{t} \in$ $\mathbf{C}^{N}$, and, for $j=1, \ldots, N$,

$$
\operatorname{ess} \sup _{|x| \geq A, x \in \Omega_{j}}\left|z_{j}(x)-\lambda_{j}\right| \rightarrow 0
$$


as $A \rightarrow \infty$, then

$$
I-K_{\lambda}, I-K_{z} \text { injective } \Rightarrow I-K_{z} \text { surjective, }\left(I-K_{z}\right)^{-1} \in B(Y) .
$$

\section{Appendix B. Guided waves.}

By a guided wave we mean a solution of the homogeneous problem which has its energy localized in or near the layer $E_{B}$. Precisely, for $a<b, S \subset \mathbf{R}^{2}$, let $S(a, b)=\left\{x \in S \mid a<x_{1}<b\right\}$. Then our definition is as follows.

Definition B.1. Call $v \in C^{1}(\bar{U})$ a guided wave if $v \not \equiv 0, v$ satisfies (1) and (2), $v$ is bounded in $E_{h}$ for every $h>0$,

$$
\sup _{n \in \mathbf{Z}} \int_{U(n, n+1)}\left(|v|^{2}+|\nabla v|^{2}\right) d x<\infty
$$

and

$$
c_{h}:=\sup _{n \in \mathbf{Z}} \int_{U_{h}(n, n+1)}\left(|v|^{2}+|\nabla v|^{2}\right) d x \rightarrow 0
$$

as $h \rightarrow \infty$.

Remark B.1. In the case when the scatterer is a diffraction grating, i.e., $k$ is periodic in the $x_{1}$-direction with some period $L$, it is usual to assume that $v$ is correspondingly quasi-periodic (i.e., that $v(x) \exp \left(-i k_{+} \cos \theta x_{1}\right)$ is periodic). Then (B.1) and (B.2) reduce to the condition that

$$
\int_{U(0, L)}\left(|v|^{2}+|\nabla v|^{2}\right) d x<\infty
$$

i.e., that the energy is finite in a single period of the grating.

Remark B.2. Conditions (B.1) and (B.2) are satisfied if $v$ decreases sufficiently rapidly in the vertical direction, in particular if, for some constants $C>0$ and $p>1 / 2$,

$$
|v(x)| \leq C\left(1+x_{2}\right)^{-p}, \quad x \in U .
$$

The following result is shown in [6].

THEOREM B.2. If $v$ is a guided wave, then $v$ satisfies the UPRC.

\section{REFERENCES}

[1] K. Attenborough, Acoustical impedance models for outdoor ground surfaces, J. Sound Vibration, 99 (1985), pp. 521-544.

[2] G. BaO, D. C. Dobson, And J. A. Cox, Mathematical studies in rigorous grating theory, J. Opt. Soc. Amer. A, 12 (1995), pp. 1029-1042.

[3] S. N. Chandler-Wilde, Boundary value problems for the Helmholtz equation in a half-plane, in Proc. 3rd Int. Conf. on Mathematical and Numerical Aspects of Wave Propagation, G. Cohen, ed., SIAM, Philadelphia, 1995, pp. 188-197.

[4] S. N. ChANDler-Wilde, The impedance boundary value problem for the Helmholtz equation in a half-plane, Math. Methods Appl. Sci., 20 (1997), pp. 813-840.

[5] S. N. Chandler-Wilde And C. R. Ross, Scattering by rough surfaces: The Dirichlet problem for the Helmholtz equation in a non-locally perturbed half-plane, Math. Methods Appl. Sci., 19 (1996), pp. 959-976.

[6] S. N. Chandler-Wilde And Bo Zhang, Electromagnetic scattering by an inhomogeneous conducting or dielectric layer on a perfectly conducting plate, Proc. Roy. Soc. London Ser. A, 454 (1998), pp. 519-542. 
[7] S. N. Chandler-Wilde and Bo Zhang, A uniqueness result for scattering by infinite rough surfaces, SIAM J. Appl. Math., 58 (1998), pp. 1774-1790.

[8] S. N. Chandler-Wilde And Bo Zhang, On the solvability of a class of second kind integral equations on unbounded domains, J. Math. Anal. Appl., 214 (1997), pp. 482-502.

[9] S. N. Chandler-Wilde And Bo Zhang, A generalised collectively compact operator theory with an application to integral equations on unbounded domains, submitted.

[10] S. N. Chandler-Wilde and D. C. Hothersall, On the Green's Function for Two-dimensional Acoustic Sound Propagation Above a Homogeneous Impedance Plane, Research Report, Dept. of Civil Engineering, University of Bradford, 1991.

[11] S. N. Chandler-Wilde And D. C. Hothersall, Efficient calculation of the Green function for acoustic propagation above a homogeneous impedance plane, J. Sound Vibration, 180 (1995), pp. 705-724.

[12] X. Chen And A. Friedman, Maxwell's equations in a periodic structure, Trans. Amer. Math. Soc., 323 (1991), pp. 465-507.

[13] D. Colton And R. Kress, Integral Equation Methods in Scattering Theory, Wiley, New York, 1983.

[14] D. Colton And R. Kress, Inverse Acoustic and Electromagnetic Scattering Theory, Springer, Berlin, 1992.

[15] J. A. DeSAnto, Exact spectral formalism for rough-surface scattering, J. Opt. Soc. Amer. A, 2 (1985), pp. 2202-2207.

[16] J.A. DeSanto and P. A. Martin, On the derivation of boundary integral equations for scattering by an infinite one-dimensional rough surface, J. Acoust. Soc. Am., 102 (1997), pp. $67-77$.

[17] C. L. DeVito, Functional Analysis, Academic Press, New York, 1978.

[18] D. Dobson and A. Friedman, The time-harmonic Maxwell equations in a doubly periodic structure, J. Math. Anal. Appl., 166 (1992), pp. 507-528.

[19] D. Gilbarg and N. S. Trudinger, Elliptic Partial Differential Equations of Second Order, 2nd ed., Springer, Berlin, 1983.

[20] A. Kirsch, Diffraction by periodic structures, in Inverse Problems in Mathematical Physics, L. Paivarinta and E. Somersalo, eds., Springer, Berlin, 1993, pp. 87-102.

[21] A. KIRSCH, An inverse problem for periodic structures, in Inverse Scattering and Potential Problems in Mathematical Physics, R. E. Kleinman, R. Kress, and E. Martensen, eds., Peter Lang, Frankfurt, 1995, pp. 75-93.

[22] J. C. Nedelec And F. Starling, Integral equation methods in a quasi-periodic diffraction problem for the time-harmonic Maxwell's equations, SIAM J. Math. Anal., 22 (1991), pp. 1679-1701.

[23] T. L. Richards and K. Attenborough, Accurate FFT-Based Hankel transforms for predictions of outdoor sound propagation, J. Sound Vibration, 109 (1986), pp. 157-167.

[24] J. S. Robertson, W. L. Siegmann, and M. J. Jacobson, Low frequency sound-propagation modeling over a locally reacting boundary with the parabolic approximation, J. Acoust. Soc. Am., 98 (1995), pp. 1130-1137.

[25] B. Szemberg, An Acoustic Scattering Problem for a Periodic, Inhomogeneous Medium, Preprint 167, Institut für Angewandte Mathematik, Universität Erlangen-Nürnberg, 1996.

[26] Y. XU, Radiation condition and scattering problem for time-harmonic acoustic waves in a stratified medium with a nonstratified inhomogeneity, IMA J. Appl. Math., 54 (1995), pp. 9-29. 